PROCEEDINGS OF THE

AMERICAN MATHEMATICAL SOCIETY

Volume 126, Number 4, April 1998, Pages 1043-1047

S 0002-9939(98)04296-8

\title{
REMARKS ON THE RESULTS BY KOSKELA CONCERNING THE RADIAL UNIQUENESS FOR SOBOLEV FUNCTIONS
}

\author{
YOSHIHIRO MIZUTA
}

(Communicated by Albert Baernstein II)

\begin{abstract}
In this note we aim to complete the results by Koskela concerning the radial uniqueness for Sobolev functions.

Let $\varphi$ be a positive nonincreasing function on the interval $(0, \infty)$, and let $\mathbf{B}$ denote the unit ball of $R^{n}$. Consider a $p$-precise function $u$ on $\mathbf{B}$ such that

$$
\int_{U(\varepsilon)}|\nabla u(x)|^{p} d x \leq \varepsilon^{p} \varphi(\varepsilon) \quad \text { for any } \varepsilon>0,
$$

where $U(\varepsilon)=\{x \in \mathbf{B}:|u(x)|<\varepsilon\}$. We give conditions on $\varphi$ which assure that $u=0$ whenever $u$ has vanishing fine boundary limits on a set of positive $p$-capacity.

We are also concerned with the sharpness.
\end{abstract}

\section{Statement of Results}

For $1<p<\infty$ and an open set $G \subset R^{n}$, we denote by $W^{1, p}(G)$ the Sobolev space of all functions $u$ on $G$ such that

$$
\int_{G}|\nabla u(x)|^{p} d x<\infty
$$

where $\nabla$ denotes the gradient. For a set $E \subset G$, we define

$$
C_{p}(E ; G)=\inf \int_{G}|\nabla u(x)|^{p} d x,
$$

where the infimum is taken over all $u \in W^{1, p}\left(R^{n}\right)$ such that $u=0$ outside $G$ and

$$
\int|x-y|^{1-n}|\nabla u(y)| d y \geqq 1 \quad \text { for every } x \in E
$$

(see [2], [7] and [9]). We write $C_{p}(E)=0$ if $C_{p}(E \cap G ; G)=0$ for every bounded open set $G$.

Let $\mathbf{B}$ denote the unit ball in $R^{n}$. For each $u \in W^{1, p}(\mathbf{B})$, we can find an extension $u^{*} \in W^{1, p}\left(R^{n}\right)$ with compact support such that

$$
u=u^{*} \quad \text { for almost every } x \in \mathbf{B}
$$

Received by the editors September 18, 1996.

1991 Mathematics Subject Classification. Primary 31B25, 31B15, 46E35.

Key words and phrases. p-precise functions, Sobolev functions, capacity, fine boundary limits.

(C)1998 American Mathematical Society 
and $u^{*}$ is $p$-precise in the sense of Ziemer [10]. In view of [4], [5], we note further that $u^{*}$ can be written as

$$
u^{*}(x)=c \int \frac{x-y}{|x-y|^{n}} \cdot \nabla u^{*}(y) d y
$$

with a constant $c$, where "." denotes the usual inner product and the integrals are absolutely convergent $p$-q.e. (quasi everywhere) on $R^{n}$, that is, for every $x \in R^{n}-E$ with $C_{p}(E)=0$. This implies that $u \in W^{1, p}(\mathbf{B})$ has a fine boundary limit $u^{*}(\xi)$ for $p$-q.e. $\xi \in \partial \mathbf{B}$ (see [3], [7] and [11]).

Our aim in this note is to show the following theorem.

Theorem 1. Let $\varphi$ be a positive nonincreasing function on the interval $(0, \infty)$ satisfying

$$
A^{-1} \varphi(r) \leq \varphi\left(r^{2}\right) \leq A \varphi(r) \quad \text { for every } r>0
$$

with a positive constant $A$ and

$$
\int_{0}^{1}[\varphi(r)]^{-1 /(p-1)} r^{-1} d r=\infty, \quad 1<p<\infty .
$$

Let $u$ be a Sobolev function in $W^{1, p}(\mathbf{B})$ such that

$$
\int_{U(\varepsilon)}|\nabla u(x)|^{p} d x \leq \varepsilon^{p} \varphi(\varepsilon) \quad \text { for any } \varepsilon>0,
$$

where $U(\varepsilon)=\{x \in \mathbf{B}:|u(x)|<\varepsilon\}$. If there exists $E \subset \partial \mathbf{B}$ such that $C_{p}(E)>0$ and the p-precise extension $u^{*}$ vanishes on $E$, then $u=0$.

Remark. P. Koskela [1] has recently treated the case where

$$
\begin{aligned}
\varphi(r)= & {[\log (1 / r)]^{p-1}, \quad[\log (1 / r)]^{p-1}[\log \log (1 / r)]^{p-1}, } \\
& {[\log (1 / r)]^{p-1}[\log \log (1 / r)]^{p-1}[\log \log \log (1 / r)]^{p-1}, \ldots }
\end{aligned}
$$

for small $r$; these functions clearly satisfy (2).

Next we show that our result is sharp in the following sense.

Theorem 2. Let $\varphi$ be a positive nonincreasing function on the interval $(0, \infty)$ satisfying (1) and

$$
\int_{0}^{1}[\varphi(r)]^{-1 /(p-1)} r^{-1} d r<\infty, \quad 1<p<\infty .
$$

Then there exists a continuous function $u$ on $R^{n}$ such that $u \in W^{1, p}\left(R^{n}\right)$, u satisfies (3), $u>0$ on $\mathbf{B}$ and $u=0$ outside $\mathbf{B}$.

\section{Proof of Theorem 1}

We use the notation $B(x, r)$ to denote the open ball centered at $x$ with radius $r$; hence $\mathbf{B}=B(0,1)$.

Let us begin with the following lemma, which is an easy consequence of the definition of $C_{p}$.

Lemma 1. Let $1<p<\infty$. For $f \in L^{p}\left(R^{n}\right)$, set

$$
E_{f}=\left\{x \in R^{n}: \int_{B(x, 1)}|x-y|^{1-n}|f(y)| d y=\infty\right\} .
$$

Then $C_{p}\left(E_{f}\right)=0$ (see e.g. [7, Proposition 5.1.1 and Theorem 6.8.2]). 
Next we prepare the following technical lemma.

Lemma 2. Let $\varphi$ be as in Theorem 1. Then there exists a positive nondecreasing function a on $(0, \infty)$ satisfying $(1)$ and such that

$$
\int_{0}^{1} a(r) r^{-1} d r=\infty
$$

and

$$
\int_{0}^{1} a(r)^{p} \varphi(r) r^{-1} d r<\infty .
$$

In fact, it suffices to consider

$$
a(r)=[\varphi(r)]^{-1 /(p-1)}\left(\int_{r}^{2}[\varphi(t)]^{-1 /(p-1)} t^{-1} d t\right)^{-1}
$$

for $r \leq 1$; set $a(r)=a(1)$ for $r>1$.

Throughout this note, let $M$ denote various constants independent of the variables in question.

Now we are ready to prove Theorem 1 . For this purpose, we may assume that $u \geqq 0$ a.e. on $\mathbf{B}$. We suppose that $\{x \in \mathbf{B}: u(x)>0\}$ has positive measure, and obtain a contradiction.

For functions $f$ and $g$, define

$$
f^{+}(x)=\max \{f(x), 0\}
$$

and

$$
f \wedge g(x)=\min \{f(x), g(x)\} .
$$

For each positive integer $j$, set

$$
u_{j}(x)=2^{j}\left[\left(u-2^{-j}\right)^{+} \wedge 2^{-j}\right]
$$

and

$$
v=\sum_{j=1}^{\infty} a_{j} u_{j} \quad \text { with } a_{j}=a\left(2^{-j}\right)
$$

for the function $a$ in Lemma 2. Note that

$$
\left|\nabla u_{j}\right|=2^{j}|\nabla u| \quad \text { a.e. on } G_{j}=\left\{x: 2^{-j}<u(x)<2^{-j+1}\right\}
$$

and

$$
\left|\nabla u_{j}\right|=0 \quad \text { a.e. outside } G_{j} .
$$

Hence it follows from (3), (1) and (6) that

$$
\begin{aligned}
\int|\nabla v|^{p} d x & =\sum_{j=1}^{\infty}\left[a_{j} 2^{j}\right]^{p} \int_{G_{j}}|\nabla u|^{p} d x \\
& \leq \sum_{j=1}^{\infty}\left[a_{j} 2^{j}\right]^{p}\left[2^{-j+1}\right]^{p} \varphi\left(2^{-j+1}\right) \\
& \leq M \sum_{j=1}^{\infty} a_{j}^{p} \varphi\left(2^{-j}\right) \\
& \leq M \int_{0}^{1} a(r)^{p} \varphi(r) r^{-1} d r<\infty .
\end{aligned}
$$


Consider $p$-precise extensions $u^{*}, v^{*} \in W^{1, p}\left(R^{n}\right)$ with compact support such that

$$
u=u^{*} \quad \text { and } \quad v=v^{*}
$$

for almost every $x \in \mathbf{B}$. If $\xi \in \partial \mathbf{B}$ and

$$
\int|\xi-y|^{1-n}\left|\nabla u^{*}(y)\right| d y<\infty,
$$

then $u^{*}(x+r(\xi-x))$ is absolutely continuous on $(0, \infty)$ for almost every $x \in \mathbf{B}$ (see $[4$, p.725] and $[8]$ ).

If $u^{*}(x)>0, u^{*}(\xi)=0$ and $f(r)=v^{*}(x+r(\xi-x))$ is absolutely continuous on $[0,1]$, then the line $L(x, \xi)=\{x+r(\xi-x): r \in[0,1]\}$ meets with $G_{j}=\{x \in \mathbf{B}$ : $\left.2^{-j}<u^{*}(x)<2^{-j+1}\right\}$ for large $j$, say $j \geqq j_{0}$, so that

$$
\int_{0}^{1}\left|f^{\prime}(r)\right| d r \geqq \sum_{j \geqq j_{0}} a_{j} \geqq M \int_{0}^{2^{-j_{0}}} a(r) r^{-1} d r=\infty
$$

because of (5). This implies that

$$
\int_{\mathbf{B}}|\xi-y|^{1-n}\left|\nabla v^{*}(y)\right| d y=\infty,
$$

which gives a contradiction by Lemma 1 , since $C_{p}(E)>0$ and $v^{*}$ is $p$-precise on $R^{n}$.

\section{Proof of Theorem 2}

Suppose

$$
\int_{0}^{1}[\varphi(r)]^{-1 /(p-1)} r^{-1} d r<\infty
$$

and set

$$
r=f(t) \equiv A \int_{0}^{t}[\varphi(s)]^{-1 /(p-1)} s^{-1} d s
$$

for a positive constant $A$. Note here that if $b>0$, then

$$
s^{b} \varphi(s) \leq M(b) t^{b} \varphi(t) \quad \text { whenever } t>s>0
$$

with a positive constant $M(b)$ (see e.g. $[6,(\varphi 5)]$ ).

We now consider the inverse function $t=f^{-1}(r)$ and define

$$
u(x)=f^{-1}(1-|x|)
$$

for $x \in \mathbf{B}$; define $u=0$ outside $\mathbf{B}$. In what follows we determine $A$ for which $u$ satisfies (3).

For small $\varepsilon>0$, find $\delta>0$ such that

$$
\varepsilon=f^{-1}(\delta) .
$$


Then we have by (7)

$$
\begin{aligned}
\int_{\{x \in \mathbf{B}:|u(x)|<\varepsilon\}}|\nabla u(x)|^{p} d x & \leq M \int_{0}^{\delta}\left|\left(f^{-1}\right)^{\prime}(r)\right|^{p} d r \\
& =M \int_{0}^{\varepsilon}\left|\frac{d r}{d t}\right|^{-(p-1)} d t \\
& =A^{1-p} M \int_{0}^{\varepsilon} t^{p-1} \varphi(t) d t \\
& \leq A^{1-p} M \varepsilon^{p} \varphi(\varepsilon) .
\end{aligned}
$$

Hence it suffices to take $A$ such that $A^{1-p} M=1$.

\section{REFERENCES}

[1] P. Koskela, A radial uniqueness theorem for Sobolev functions, Bull. London Math. Soc. 27 (1995), 460-466. MR 96e:31010

[2] N. G. Meyers, A theory of capacities for potentials in Lebesgue classes, Math. Scand. 26 (1970), 255-292. MR 43:3474

[3] N. G. Meyers, Taylor expansion of Bessel potentials, Indiana Univ. Math. J. 23 (1974), 1043-1049. MR 50:980

[4] Y. Mizuta, Existence of various boundary limits of Beppo Levi functions of higher order, Hiroshima Math. J. 9 (1979), 717-745. MR 81d:31013

[5] Y. Mizuta, Boundary behavior of $p$-precise functions on a half space of $R^{n}$, Hiroshima Math. J. 18 (1988), 73-94. MR 89d:31014

[6] Y. Mizuta, Continuity properties of potentials and Beppo-Levi-Deny functions, Hiroshima Math. J. 23 (1993), 79-153. MR 94d:31005

[7] Y. Mizuta, Potential theory in Euclidean spaces, Gakkōtosyo, Tokyo, 1996. CMP 97:06

[8] M. Ohtsuka, Extremal length and precise functions in 3-space, Lecture Notes, Hiroshima University, 1973.

[9] Yu. G. Reshetnyak, The concept of capacity in the theory of functions with generalized derivatives, Siberian Math. J. 10 (1969), 818-842. MR 43:2234

[10] W. P. Ziemer, Extremal length as a capacity, Michigan Math. J. 17 (1969), 117-128. MR 42:3299

[11] W. P. Ziemer, Weakly differentiable functions, Springer-Verlag, New York, 1989. MR 91e:46046

The Division of Mathematical and Information Sciences, Faculty of Integrated Arts and Sciences, Hiroshima University, Higashi-Hiroshima 739, Japan

E-mail address: mizuta@mis.hiroshima-u.ac.jp 